\title{
Patterns in matchings and rook placements
}

\author{
Jonathan Bloom and Sergi Elizalde $\|^{\dagger}$ \\ Department of Mathematics, Dartmouth College, Hanover, NH, USA
}

\begin{abstract}
Extending the notion of pattern avoidance in permutations, we study matchings and set partitions whose arc diagram representation avoids a given configuration of three arcs. These configurations, which generalize 3 -crossings and 3-nestings, have an interpretation, in the case of matchings, in terms of patterns in full rook placements on Ferrers boards. We enumerate 312-avoiding matchings and partitions, obtaining algebraic generating functions, unlike in the 321-avoiding (i.e., 3-noncrossing) case. Our approach also provides a more direct proof of a formula of Bóna for the number of 1342-avoiding permutations. Additionally, we give a bijection proving the shape-Wilf-equivalence of the patterns 321 and 213 which simplifies existing proofs by Backelin-West-Xin and Jelínek.

Résumé. Étendant la notion de motifs exclus dans des permutations, nous étudions des appariements et partitions dont le diagramme d'arc évite une configuration donnée de trois arcs. Ces configurations, qui généralisent les 3croissements et les 3-emboîtements, ont une interprétation, dans le cas d'appariements, en termes de motifs dans des placements pleins de tours sur des tables de Ferrers. Nous énumérons les appariements et les partitions qui évitent 312 , obtenant des séries génératrices algébriques, contrairement au cas du motif 321 . Notre approche fournit aussi une démonstration plus directe d'une formule de Bóna pour le nombre de permutations qui évitent 1342. En plus, nous donnons une preuve bijective de l'équivalence au sens de la forme et de Wilf des motifs 321 et 213 qui simplifie les preuves de Backelin-West-Xin et Jelínek.
\end{abstract}

Keywords: matching, set partition, bijection, pattern avoidance, shape-Wilf-equivalence, rook placement, Dyck path.

\section{Introduction}

Pattern avoidance in matchings is a natural extension of pattern avoidance in permutations. Indeed, a permutation of $[n]=\{1,2, \ldots, n\}$ can be thought of as matching of $[2 n]$ where each element of $[n]$ is paired up with an element of $[2 n] \backslash[n]$. The natural translation of the definition of patterns in permutations to this type of matchings extends to all perfect matchings, and more generally, to set partitions - which, when all the blocks have size 2, are just perfect matchings. We will use the term matching to refer to a perfect matching, when it creates no confusion. On the other hand, the well-studied notions of $k$-crossings and $k$-nestings in matchings and set partitions, in our language, are simply occurrences of the patterns $k \ldots 21$ and $12 \ldots k$, respectively. Additionally, by viewing matchings as certain fillings of Ferrers boards, patterns in matchings relate to patterns in Ferrers boards, and thus to the concept of shape-Wilf-equivalence of permutations.

\footnotetext{
$\dagger$ Partially supported by NSF grant DMS-1001046.

1365-8050 (c) 2013 Discrete Mathematics and Theoretical Computer Science (DMTCS), Nancy, France
} 
Motivated by these connections and by the recent work on crossings, nestings, permutation patterns, and shape-Wilf-equivalence, we study matchings and partitions that avoid patterns of length 3 . We consolidate and simplify recent work on the classification of these patterns, and we obtain new results on the enumeration of matchings and partitions that avoid some of these patterns.

\subsection{Background}

We represent a matching of $[2 n]$ as an arc diagram as follows: place $2 n$ equally spaced points on a horizontal line, numbered from left to right, and draw an arc between the two vertices of each of the $n$ pairs. The picture on the left of Fig. 2 corresponds to the matching $(1,6),(2,12),(3,4),(5,7),(8,10),(9,11)$. If $i<j<k<\ell$, two $\operatorname{arcs}(i, k),(j, \ell)$ form a crossing, and two $\operatorname{arcs}(i, \ell),(j, k)$ form a nesting. Similarly, a partition of $[n]$ is represented by drawing, for each block $\left\{i_{1}, i_{2}, \ldots, i_{a}\right\}$ of size $a$ with $i_{1}<i_{2}<\cdots<i_{a}$, $a-1 \operatorname{arcs}\left(i_{1}, i_{2}\right),\left(i_{2}, i_{3}\right), \ldots,\left(i_{a-1}, i_{a}\right)$. A crossing in the partition is then a pair of $\operatorname{arcs}(i, k),(j, \ell)$, and a nesting is a pair of arcs $(i, \ell),(j, k)$, where $i<j<k<\ell$.

Crossings and nestings in matchings and partitions have been studied for decades. It is well known that the number of perfect matchings on $[2 n]$ with no crossings (or with no nestings) is the $n$-th Catalan number $C_{n}$, which also equals the number of partitions of $[n]$ of with no crossings, and the number of those with no nestings. More generally, attention has focused on the study of $k$-crossings ( $k$-nestings), which are sets of $k$ pairwise crossing (respectively, nesting) arcs. For set partitions, the above definition, which we use throughout the paper, is the same given by Chen, Deng, Du, Stanley and Yan [7] and Krattenthaler [16]. However, we point out that different definitions of pattern avoidance for partitions have been introduced by Klazar [14] and Sagan [17].

The number of 3-nonnesting matchings of $[2 n]$ (viewed as fixed-point-free involutions with no decreasing sequence of length 6) was found by Gouyou-Beauchamps [12], who recursively constructed a bijection onto pairs of noncrossing Dyck paths with $2 n$ steps, counted by $C_{n} C_{n+2}-C_{n+1}^{2}$. More recently, Chen et al. [7] showed that the number of $k$-noncrossing matchings (i.e., containing no $k$-crossing) of [2n] equals the number of $k$-nonnesting (i.e., containing no $k$-nesting) ones, for all $k$, and that the analogous results for partitions hold as well. Their proof, which uses vacillating tableaux and a variation of RobinsonSchensted insertion and deletion, also provides a bijection between $k$-noncrossing matchings and certain $(k-1)$-dimensional closed lattice walks, from where a determinant formula for the generating function in terms of hyperbolic Bessel functions follows.

Less is known about the enumeration of $k$-noncrossing set partitions. Bousquet-Mélou and Xin [5] settled the case $k=3$ using a bijection into lattice paths to derive a functional equation for the generating function, which then is solved by the kernel method. They showed that the generating function for 3noncrossing set partitions is D-finite, that is, it satisfies a linear differential equation with polynomial coefficients. This is conjectured not to be the case for $k>3$. For $k$-nonnesting set partitions, additional functional equations for the generating functions have been obtained by Burrill et al. [6] using generating trees for open arc diagrams.

By interpreting matchings and partitions as rook placements on Ferrers boards and using the growth diagram construction of Fomin, Krattenthaler [16] gave a simpler description of the bijections in [7] proving the symmetry of crossing and nesting number on matchings and partitions. He extended the results to fillings of Ferrers boards with nonnegative integers. Other extensions have been given by de Mier [9] to fillings with prescribed row and column sums.

As mentioned before, $k$-crossings (respectively, $k$-nestings) in matchings have a simple interpretation as occurrences of the monotone decreasing (respectively, increasing) pattern of length $k$. In this paper we 
study and enumerate matchings that avoid other patterns of length 3 , and in some cases, we extend our results to the enumeration of pattern-avoiding partitions. The translation of crossings and nestings to the language of permutation patterns becomes natural via a bijection between matchings and certain fillings of Ferrers boards, called full rook placements, described in Section 2.2. For such fillings, the definitions of pattern containment and avoidance in permutations generalize routinely, and they have been widely studied in the literature. In this setting, Stankova and West [19] introduced the concept of shape-Wilfequivalence, and they showed that the patterns 231 and 312 are shape-Wilf-equivalent. A simpler proof of this fact was later given by Bloom and Saracino [3]. As we will see, if two patterns are shape-Wilfequivalent, then the number of matchings avoiding one is the same as the number of those avoiding the other, and the same is true for partitions. Backelin, West and Xin [1] showed that $12 \ldots k$ and $k \ldots 21$ are shape-Wilf-equivalent. A more direct proof of their result, which implies again that $k$-nonnesting and $k$-noncrossing matchings are equinumerous, was given by Krattenthaler [16]. It also follows from [1] that 123 and 213 are shape-Wilf-equivalent. Thus, there are three shape-Wilf-equivalence classes of patterns of length 3, namely $123 \sim 321 \sim 213,231 \sim 312$, and 132 .

Jelínek [13] reproved some of these results independently in the context of matchings, by giving bijections between 231-avoiding matchings and 312-avoiding ones, and between 213-avoiding matchings and 123 -avoiding (i.e. 3-nonnesting) ones.

Finally, let us mention that Stankova [18] compared, for each one of the three shape-Wilf-equivalence classes of patterns of length 3, the number of full rook placements on any given Ferrers board avoiding each a pattern in the class. She showed that the number of 231-avoiding placements is no larger than the number of 321-avoiding placements (this is also proved in [13]), which is in turn no larger than the number of 132 -avoiding ones.

\subsection{Structure of the paper}

In Section 2 we define patterns in matchings, in set partitions, and in rook placements on Ferrers boards, and we set the notation for the rest of the paper. In Sections 3 and 4 we study two of the three shape-Wilfequivalence classes of patterns of length 3. In Section 3 we give a new simple bijection between 123avoiding matchings and 213-avoiding ones, as well as an extension of work of Gouyou-Beauchamps [12] for matchings with fixed points (i.e., not necessarily perfect). In Section 4 we enumerate 231 -avoiding (equivalently, 312-avoiding) matchings and partitions, and we show that their generating functions are algebraic, in contrast to the case of 123-avoiding matchings [12] and partitions [5]. We then use our techniques for matchings to obtain a new proof of Bóna's formula enumerating 1342-avoiding permutations [4]. This leaves one pattern of length 3 , namely 132, for which we have been unable to find a formula for the number of 132-avoiding matchings or partitions. We argue in [2] that this question is related to the outstanding open problem of enumerating 1324-avoiding permutations [4, 8]. Finally, Section 5 summarizes some results about matchings and partitions that avoid pairs of patterns of length 3 . The proofs that are omitted in this extended abstract can be found in [2].

\section{Matchings, partitions, and rook placements}

\subsection{Ferrers boards}

A Ferrers board is a left-justified array of unit squares so that the number of squares in each row is less than or equal to the number of squares in the row below. To be precise, consider an $n \times n$ array of unit 
squares in the $x y$-plane, whose bottom left corner is at the origin $(0,0)$. The vertices of the unit squares are lattice points in $\mathbb{Z}^{2}$. For any vertex $V=(a, b)$, let $\Gamma(V)$ be the set of unit squares inside the rectangle $[0, a] \times[0, b]$. Then, a subset $F$ of the $n \times n$ array with the property that $\Gamma(V) \subseteq F$ for each vertex in $F$ is a Ferrers board. Equivalently, $F$ is bounded by the coordinate lines and by a lattice path from $(0, n)$ to $(n, 0)$ with east steps $(1,0)$ and south steps $(0,-1)$. We call this path the border of $F$, and we denote its vertices by $V_{0}, \ldots, V_{2 n}$, where $V_{0}=(0, n), V_{n}=(n, 0)$ and $V_{i+1}$ is immediately below or to the right of $V_{i}$. An example of these definitions appears in [2, Fig. 1].

Definition $1 A$ full rook placement is a pair $(R, F)$ where $F$ is a Ferrers board and $R$ is a subset of squares of $F$ (marked by placing a rook in each one of them) such that each row and each column of $F$ contains exactly one rook. Let $\mathcal{R}_{F}$ denote the set of full rook placements on $F$.

In this paper, the term placement will always refer to a full rook placement. For a Ferrers board $F$ to admit a full rook placement, the number or nonempty rows must equal the number of nonempty columns, and the coordinates $(x, y)$ of the vertices in the border of $F$ must satisfy $x \geq y$. We denote by $\mathcal{F}_{n}$ the set of Ferrers boards satisfying this condition and having $n$ nonempty rows and columns. The border of $F \in \mathcal{F}_{n}$, which we denote by $D_{F}$, is a lattice path from $(0, n)$ to $(n, 0)$ with steps east $(e=(1,0))$ and south $(s=(0,-1))$ that remains above the line $y=n-x$. We denote by $\mathcal{D}_{n}$ the set of such paths, which we call Dyck paths of semilength $n$ (despite being rotated from other standard ways of drawing them). The map $F \mapsto D_{F}$ is a trivial bijection between $\mathcal{F}_{n}$ and $\mathcal{D}_{n}$. A peak on a Dyck path is an occurrence of $e s$ (as consecutive steps), and a valley is an occurrence of $s e$.

We let

$$
\mathcal{R}_{n}=\bigcup_{F \in \mathcal{F}_{n}} \mathcal{R}_{F}
$$

be the set of all placements on boards in $\mathcal{F}_{n}$. Denote by $\mathcal{S}_{n}$ the set of permutations of $\{1,2, \ldots, n\}$. To each full rook placement $(R, F)$ where $F \in \mathcal{F}_{n}$, one can associate a permutation $\pi_{R} \in \mathcal{S}_{n}$ by letting $\pi_{R}(i)=j$ if $R$ has a rook in column $i$ and row $j$ (our convention is to number the columns of $F$ from left to right and its rows from bottom to top, as in the usual cartesian coordinates). In the case that $F \in \mathcal{F}_{n}$ is the square Ferrers board, this map is a bijection between $\mathcal{R}_{F}$ and $\mathcal{S}_{n}$. More generally, given a vertex $V$ of the border of $F$, the restriction of the placement $R$ to the rectangle $\Gamma(V)$, which consists of the squares $R \cap \Gamma(V)$, determines a unique permutation in $\mathcal{S}_{k}$, where $k=|R \cap \Gamma(V)|$. This permutation is obtained by disregarding empty rows and columns, and then applying the above map. Under this correspondence it makes sense to consider concepts such as the longest increasing sequence in $R \cap \Gamma(V)$.

Recall that a permutation $\pi \in \mathcal{S}_{n}$ avoids another permutation $\tau \in \mathcal{S}_{k}$ (usually called a pattern) if there is no subsequence $\pi\left(i_{1}\right) \ldots \pi\left(i_{k}\right)$ with $i_{1}<\ldots<i_{k}$ that is order-isomorphic to $\tau(1) \ldots \tau(k)$. The number of $\tau$-avoiding permutations in $\mathcal{S}_{n}$ is denoted by $\mathcal{S}_{n}(\tau)$. Viewing permutations as full rook placements on the square Ferrers board, $\pi$ avoids $\tau$ if the placement corresponding to $\tau$ cannot be obtained from the placement corresponding to $\pi$ by removing rows and columns. This definition has been generalized [1] to rook placements as follows.

Definition 2 A full rook placement $(R, F)$ avoids $\tau \in \mathcal{S}_{k}$ if and only if for every vertex $V$ on the border of $F$, the permutation given by $R \cap \Gamma(V)$ avoids $\tau$. Let $\mathcal{R}_{F}(\tau)$ be the set of full rook placements on $F$ that avoid $\tau$. Similarly, let

$$
\mathcal{R}_{n}(\tau)=\bigcup_{F \in \mathcal{F}_{n}} \mathcal{R}_{F}(\tau)
$$


Definition 3 Two patterns $\sigma$ and $\tau$ are said to be shape-Wilf-equivalent, denoted $\sigma \sim \tau$, iffor any Ferrers board $F$ we have $\left|\mathcal{R}_{F}(\sigma)\right|=\left|\mathcal{R}_{F}(\tau)\right|$.

Clearly, if two patterns are shape-Wilf-equivalent, then they are also Wilf-equivalent, meaning that they are avoided by the same number of permutations. The converse is not true, as shown by the fact that there is one Wilf-equivalence class for patterns of length 3 , but three shape-Wilf-equivalence classes: $123 \sim 321 \sim 213,231 \sim 312$, and 132 .

Regarding shape-Wilf-equivalence of patterns of arbitrary length, two important results are due to Backelin, West and Xin [1]. One states that $12 \ldots k \sim k \ldots 21$ for all $k$, and the other one is the following.

Proposition 2.1 ([1]) Let $\sigma, \tau \in \mathcal{S}_{k}$ and $\rho \in \mathcal{S}_{\ell}$. If $\sigma \sim \tau$, then $\sigma \rho^{\prime} \sim \tau \rho^{\prime}$, where $\rho^{\prime}$ is obtained from $\rho$ by adding $k$ to each of its entries.

Denote by $\mathcal{D}_{n}^{2}$ the set of pairs $\left(D_{0}, D_{1}\right)$ of Dyck paths $D_{0}, D_{1} \in \mathcal{D}_{n}$ such that $D_{0}$ never goes above $D_{1}$. We say that $D_{0}$ and $D_{1}$ are noncrossing, and we call $D_{0}$ the bottom path and $D_{1}$ the top path. For any $F \in \mathcal{F}_{n}$, we denote by $\mathcal{D}_{F}^{2}$ the set of pairs $\left(D_{0}, D_{F}\right) \in \mathcal{D}_{n}^{2}$, that is, those where the top path is the border of $F$.

\subsection{Matchings}

Denote by $\mathcal{M}_{n}$ the set of perfect matchings on $[2 n]$. If $(i, j)$ is a matched pair with $i<j$, we call $i$ an opener and $j$ a closer. The following natural bijection between $\mathcal{M}_{n}$ and $\mathcal{R}_{n}$, which we denote $\kappa$, has been used in [9, 13]. Given a matching $M \in \mathcal{M}_{n}$, construct a path from $(0, n)$ to $(n, 0)$ by reading the vertices of $M$ in increasing order, and adding an east step for each opener, and a south step for each closer. This path is clearly a Dyck path, so it is the border of a Ferrers board $F \in \mathcal{F}_{n}$, which we call the shape of $M$. Each column of $F$ is naturally associated to an opener of $M$ (the vertex that produced the east step at the top of the column), and similarly each row is naturally associated to a closer. Now define a full rook placement on $F$ by placing a rook in the column associated to $i$ and the row associated to $j$ for each matched pair $(i, j)$. Two examples of the bijection $\kappa$ are given in Fig. 2 For fixed $F \in \mathcal{F}_{n}$, denote by $\mathcal{M}_{F}=\kappa^{-1}\left(\mathcal{R}_{F}\right)$ the set of matchings of shape $F$. Note that $\mathcal{M}_{n}=\bigcup_{F \in \mathcal{F}_{n}} \mathcal{M}_{F}$. In light of this bijection, the definition of pattern avoidance in Ferrers boards translates naturally to matchings.

Definition 4 We say that a matching $M \in \mathcal{M}_{n}$ avoids the pattern $\tau \in \mathcal{S}_{k}$ if the corresponding rook placement $\kappa(M)$ does. Equivalently, $M$ avoids $\tau$ if there are no $2 k$ vertices $1 \leq i_{1}<\ldots<i_{2 k} \leq n$ such that $M$ contains all the pairs $\left(i_{a}, i_{2 k+1-\tau(a)}\right)$ for $1 \leq a \leq k$. Let $\mathcal{M}_{F}(\tau)=\kappa^{-1}\left(\mathcal{R}_{F}(\tau)\right)$ be the set of $\tau$-avoiding matchings of shape $F$, and let $\mathcal{M}_{n}(\tau)=\bigcup_{F \in \mathcal{F}_{n}} \mathcal{M}_{F}(\tau)$.

This definition extends the notions of $k$-noncrossing and $k$-nonnesting matchings studied in [7, 16]. Recall that a matching is $k$-noncrossing if it contains no $k$ mutually crossing arcs. In our terminology, this is equivalent to avoiding the pattern $k \ldots 21$. Similarly, a matchings is $k$-nonnesting if it contains no $k$ mutually crossing arcs, which is equivalent to avoiding $12 \ldots k$.

For patterns $\tau \in \mathcal{S}_{3}$, which are the focus of this paper, we can describe $\mathcal{M}_{n}(\tau)$ as the set of matchings $M \in \mathcal{M}_{n}$ containing no three arcs whose endpoints occur in the same order as in the corresponding configuration in Fig. 1 .

Since $\kappa$ is a bijection, it is clear that $\left|\mathcal{M}_{F}(\tau)\right|=\left|\mathcal{R}_{F}(\tau)\right|$ for any $\tau$. Thus, shape-Wilf-equivalence can be interpreted in terms of pattern-avoiding matchings: $\sigma \sim \tau$ if and only if $\left|\mathcal{M}_{F}(\sigma)\right|=\left|\mathcal{M}_{F}(\tau)\right|$ for every Ferrers board $F$. In particular, if $\sigma \sim \tau$, then $\left|\mathcal{M}_{n}(\sigma)\right|=\left|\mathcal{M}_{n}(\tau)\right|$ for all $n$. The converse 


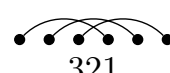

321

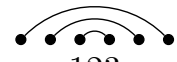

123

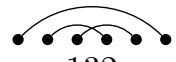

132

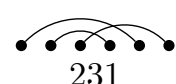

231

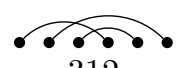

312

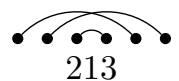

Fig. 1: Forbidden configurations corresponding to $\tau \in \mathcal{S}_{3}$.

statement is false in general. For example, it is trivial by symmetry that $\left|\mathcal{M}_{n}(2341)\right|=\left|\mathcal{M}_{n}(4123)\right|$ for all $n$, but the patterns 2341 and 4123 are not shape-Wilf-equivalent, since $\left|\mathcal{M}_{F}(2341)\right| \neq\left|\mathcal{M}_{F}(4123)\right|$ for the Ferrers boards in $\mathcal{F}_{6}$ consisting of a $6 \times 6$ square with two missing boxes.

\subsection{Set partitions}

Denote by $\mathcal{P}_{n}$ the set of partitions of $[n]$. For each block $\left\{i_{1}, i_{2}, \ldots, i_{a}\right\}$ with $i_{1}<i_{2}<\cdots<i_{a}$ and $a \geq 2$, we call $i_{1}$ an opener, $i_{a}$ a closer, and we say that $i_{2}, \ldots, i_{a-1}$ are transitory vertices. If $a=1$, the vertex $i_{1}$ is called a singleton. We will use the term partition to refer to a set partition when it creates no confusion. Note that matchings are partitions where all blocks have size 2 . The definition of pattern avoidance for matchings extends to partitions as follows.

Definition 5 We say that a partition $P \in \mathcal{P}_{n}$ avoids the pattern $\tau \in \mathcal{S}_{k}$ if there are no $2 k$ vertices $1 \leq i_{1}<\ldots<i_{2 k} \leq n$ such that $P$ contains all the arcs $\left(i_{a}, i_{2 k+1-\tau(a)}\right)$ for $1 \leq a \leq k$. Denote by $\mathcal{P}_{n}(\tau)$ the set of $\tau$-avoiding partitions of $[n]$.

Note that in the above definition, singleton blocks of $P$ do not contribute to occurrences of any pattern $\tau$.

\section{The patterns $123 \sim 321 \sim 213$}

The equivalence $123 \sim 321$ was first proved in [1], and later simplified by Chen et al [7] and by Krattenthaler [16]. The equivalence $321 \sim 213$ was proved by Backelin, West and Xin [1], and later by Jelínek [13]. In this section we provide a short bijective proof of the fact that $321 \sim 213$, greatly simplifying the proofs in [1, 13]. For the rest of this section, we fix a Ferrers board $F \in \mathcal{F}_{n}$, and we let $V_{i}$ denote the $i$ th vertex on the border of $F$.

Theorem 3.1 There are explicit bijections $\Delta_{321}: \mathcal{M}_{F}(321) \rightarrow \mathcal{D}_{F}^{2}$ and $\Delta_{213}: \mathcal{M}_{F}(213) \rightarrow \mathcal{D}_{F}^{2}$. Therefore, $321 \sim 213$.

This theorem will follow from Theorems 3.2 and 3.3 below. In a different form, the bijection $\Delta_{321}$ was constructed by Chen et al. [7] using vacillating tableaux. Here we provide a short description of this bijection in our language. Recall that matchings can be viewed as full rook placements via the bijection $\kappa: \mathcal{M}_{F} \rightarrow \mathcal{R}_{F}$ described in Section 2.2 .

It will be convenient to identify a Dyck path $D \in \mathcal{D}_{n}$ with the sequence $d_{0} d_{1} \ldots d_{2 n}$ that records the distances from its vertices to the main diagonal $y=n-x$. More precisely, if $V_{i}=(a, b)$, then $d_{i}=a+b-n$. We call $d_{0} d_{1} \ldots d_{2 n}$ the height sequence of $D$. Fix $h_{0} h_{1} \ldots h_{2 n}$ to be the height sequence of $D_{F}$.

For $(R, F) \in \mathcal{R}_{F}$, define the sequence $j_{0} \ldots j_{2 n}$ by letting $j_{i}=2 \ell_{i}-h_{i}$, where $\ell_{i}$ is the length of the longest increasing sequence in $R \cap \Gamma\left(V_{i}\right)$. A straightforward argument (see [2]) shows that $j_{0} \ldots j_{2 n}$ is a height sequence for some Dyck path, which we denote by $D_{R, F}$. Additionally, we show that $j_{i} \leq h_{i}$ for all $i$, and so $\left(D_{R, F}, D_{F}\right) \in \mathcal{D}_{F}^{2}$. Define the map $\delta_{321}: \mathcal{R}_{F}(321) \rightarrow \mathcal{D}_{F}^{2}$ by letting $\delta_{321}(R, F)=$ 
$\left(D_{R, F}, D_{F}\right)$. Then define $\Delta_{321}=\delta_{321} \circ \kappa$. The proof of following theorem is omitted in this extended abstract, but it may be found in [2].

Theorem 3.2 The map $\delta_{321}: \mathcal{R}_{F}(321) \rightarrow \mathcal{D}_{F}^{2}$ is a bijection, and thus so is $\Delta_{321}: \mathcal{M}_{F}(321) \rightarrow \mathcal{D}_{F}^{2}$.

Now we turn to the second part of the proof of Theorem 3.1. Even though a different bijection between $\mathcal{M}_{F}(213)$ and $\mathcal{D}_{F}^{2}$ has already been given by Jelínek in [13], here we present a much simpler bijection $\Delta_{213}$ through a short pictorial argument.

As in the case of 321-avoiding matchings, it is convenient to let $\Delta_{213}=\delta_{213} \circ \kappa$, where the map $\delta_{213}$ : $\mathcal{R}_{F}(213) \rightarrow \mathcal{D}_{F}^{2}$ is defined as $\delta_{213}(R, F)=\left(D, D_{F}\right)$, with $D$ given by the following construction. As the pattern 213 ends with its largest entry, the fact that $(R, F)$ is 213 -avoiding implies that $\pi_{R} \in \mathcal{S}_{n}(213)$. Let $F_{R}$ be the minimal Ferrers board that contains $R$. In a different language, the bijection between $\mathcal{S}_{n}(213)$ and $\mathcal{D}_{n}$ that sends $\pi_{R}$ to $D_{F_{R}}$ appears in [15]. We define the bottom path in $\delta_{213}(R, F)$ to be $D=D_{F_{R}}$. Note that $F_{R} \subseteq F$ by definition, so $D_{F_{R}}$ and $D_{F}$ are noncrossing Dyck paths. The following theorem is now clear.

Theorem 3.3 The map $\delta_{213}: \mathcal{R}_{F}(213) \rightarrow \mathcal{D}_{F}^{2}$ is a bijection, and thus so is $\Delta_{213}: \mathcal{M}_{F}(213) \rightarrow \mathcal{D}_{F}^{2}$.

Examples of the maps $\delta_{321}$ and $\delta_{213}$, together with the complete bijection from between $\mathcal{M}_{F}(321)$ and $\mathcal{M}_{F}(213)$, is given in Fig. 2

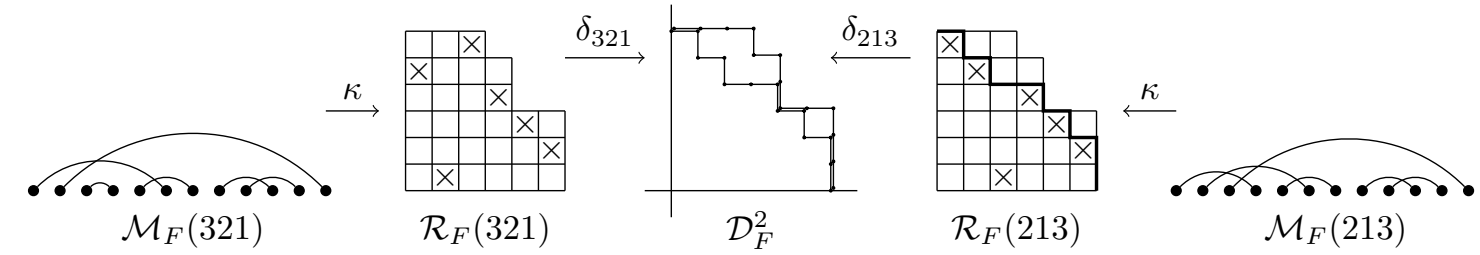

Fig. 2: An example of the bijection between $\mathcal{M}_{F}(321)$ and $\mathcal{M}_{F}(213)$. The bold path on the Ferrers board on the right represents the border of $F_{R}$.

In the particular case that $F \in \mathcal{F}_{n}$ is the square board, the composition $\Delta_{213}^{-1} \circ \Delta_{321}$ gives a bijection between $\mathcal{S}_{n}(321)$ and $\mathcal{S}_{n}(213)$ which coincides, up to symmetry, with a bijection of Elizalde and Pak [10].

We end this section by mentioning that $\Delta_{321}$ and $\Delta_{213}$ can be generalized to bijections between pattern-avoiding matchings with fixed points and pairs of noncrossing Dyck paths satisfying a certain condition. These generalizations, which we describe in the full paper [2], extend the results of GouyouBeauchamps [12] involving Young tableaux with at most 4 or 5 rows and 54321-avoiding involutions, which in our language become 123-avoiding matchings with fixed points.

\section{The patterns $231 \sim 312$}

The first proof of the equivalence $231 \sim 312$ was given by Stankova and West [19]. Later, Bloom and Saracino [3] gave a more direct proof. The main ingredient in Bloom and Saracino's construction is a bijection between 231-avoiding full rook placements of a given Ferrers board $F \in \mathcal{F}_{n}$ and certain labelings of the vertices on the border of $F$. Recall that the vertices $V_{0} V_{1} \ldots V_{2 n}$ are ordered from $(0, n)$ to $(n, 0)$. 
We define a labeled Dyck path of semilength $n$ to be a pair $(D, \alpha)$ where $D \in \mathcal{D}_{n}$, and $\alpha=$ $\alpha_{0} \alpha_{1} \ldots \alpha_{2 n}$ is an integer sequence with the following monotonicity property: if $V_{i+1}$ is to the right of $V_{i}$, then $\alpha_{i} \leq \alpha_{i+1} \leq \alpha_{i}+1$, else $\alpha_{i} \geq \alpha_{i+1} \geq \alpha_{i}-1$. We think of $\alpha_{i}$ as the label of vertex $V_{i}$.

We say that two vertices $V_{i}=\left(x_{i}, y_{i}\right)$ and $V_{j}=\left(x_{j}, y_{j}\right)$ of $D$ are aligned if $x_{i}-x_{j}=y_{i}-y_{j}$ and the line segment connecting the points $V_{i}$ and $V_{j}$ lies strictly below $D$ (except for the endpoints of the segment, which are on $D)$. We say that a labeled Dyck path $(D, \alpha)$ has the diagonal property if for any two aligned vertices $V_{i}$ and $V_{j}$ with $i<j$, we have $\alpha_{i} \geq \alpha_{j}$. We say $(D, \alpha)$ satisfies the 0 -condition if for each $i$, one has $\alpha_{i}=0$ if and only if $V_{i}$ lies on the diagonal $y=n-x$. For $F \in \mathcal{F}_{n}$, we denote by $\mathcal{L}_{F}$ the set of labelings $\left(D_{F}, \alpha\right)$ of the boundary of $F$ that satisfy both the diagonal property and the 0-condition. We also let $\mathcal{L}_{n}=\bigcup_{F \in \mathcal{F}_{n}} \mathcal{L}_{F}$.

Bloom and Saracino's bijection in [3] between placements and labeled Dyck paths is the map $\Pi$ : $\mathcal{R}_{F}(312) \rightarrow \mathcal{L}_{F}$ that sends $(R, F) \in \mathcal{R}_{F}(312)$ to the pair $\left(D_{F}, \alpha\right)$ where, for $0 \leq i \leq 2 n$, the label $\alpha_{i}$ is the length of the longest increasing sequence in $R \cap \Gamma\left(V_{i}\right)$. In a slight abuse of notation, we also denote by $\Pi$ the bijection induced by $\Pi$ from $\mathcal{R}_{n}(312)=\bigcup_{F \in \mathcal{F}_{n}} \mathcal{R}_{F}(312)$ to $\mathcal{L}_{n}=\bigcup_{F \in \mathcal{F}_{n}} \mathcal{L}_{F}$.

\subsection{2-avoiding matchings}

In this section we enumerate 312 -avoiding matchings, or equivalently, 231-avoiding ones.

Theorem 4.1 The generating function for 312-avoiding matchings is

$$
\sum_{n \geq 0}\left|\mathcal{M}_{n}(312)\right| z^{n}=\frac{54 z}{1+36 z-(1-12 z)^{3 / 2}} .
$$

The asymptotic behavior of its coefficients is given by

$$
\left|\mathcal{M}_{n}(312)\right| \sim \frac{3^{3}}{2^{5} \sqrt{\pi n^{5}}} 12^{n} .
$$

Proof: We first translate the problem into an enumeration of labeled Dyck paths. The composition $\Pi \circ \kappa$ is a bijection between $\mathcal{M}_{n}(312)$ and $\mathcal{L}_{n}$, so we have $L(z):=\sum_{n \geq 0}\left|\mathcal{M}_{n}(312)\right| z^{n}=\sum_{n>0}\left|\mathcal{L}_{n}\right| z^{n}$.

We will find an expression for $L(z)$ using the recursive structure of Dyck paths: every $D \in \mathcal{D}_{n}$ with $n \geq 1$ uniquely decomposes as $e D_{1} s D_{2}$ where $e$ is an east step, $s$ is a south step, and $D_{1}$ and $D_{2}$ are Dyck paths. Even though this decomposition can be extended to deal with labeled Dyck paths by transferring the label on each vertex of $D$ to the corresponding vertex of $e D_{1} s$ or $D_{2}$, the fact that the labels on $e D_{1} s$ satisfy the 0 -condition does not guarantee that the labels on $D_{1}$ do, even if their values are decreased by 1 .

To deal with this problem, we relax the 0 -condition and consider the larger set $\mathcal{K}_{n}$ consisting of all labeled Dyck paths $(D, \alpha)$ of semilength $n$ that have the diagonal property and satisfy $\alpha_{2 n}=0$. Let $\mathcal{K}=\bigcup_{n \geq 0} \mathcal{K}_{n}$, and denote by $K(u, z)=\sum_{n \geq 0} \sum_{(D, \alpha) \in \mathcal{K}_{n}} u^{\alpha_{0}} z^{n}$ the generating function for such paths according to the value of the first label.

To obtain an equation for $K(u, z)$, first consider the following operation: given $(A, \alpha) \in \mathcal{K}_{i},(B, \beta) \in$ $\mathcal{K}_{j}$, let $(A, \alpha) \oplus(B, \beta) \in \mathcal{K}_{i+j}$ be the concatenation of Dyck paths $A B$ with labels $\left(\alpha_{0}+\beta_{0}\right)\left(\alpha_{1}+\right.$ $\left.\beta_{0}\right) \ldots\left(\alpha_{2 i}+\beta_{0}\right) \beta_{1} \ldots \beta_{2 j}$. In other words, the labels along $A$ are increased by $\beta_{0}$, and the labels along $B$ do not change. Every nonempty $(D, \gamma) \in \mathcal{K}$ can be decomposed uniquely as $(D, \gamma)=\left(e D_{1} s, \alpha\right) \oplus$ $\left(D_{2}, \beta\right)$ where $\left(e D_{1} s, \alpha\right),\left(D_{2}, \beta\right) \in \mathcal{K}$. Whereas $\left(D_{2}, \beta\right)$ is an arbitrary element of $\mathcal{K}$, the labeling $\alpha$ of the elevated Dyck path $e D_{1} s$ can be of four different types, according to whether $\alpha_{0}=\alpha_{1}$ and whether 
$\alpha_{2 i-1}=\alpha_{2 i}$, where $i$ is the semilength of $e D_{1} s$. Analyzing these four possibilities, the decomposition translates into the functional equation

$$
K(u, z)=1+z K(u, z)\left(2 K(u, z)+u K(u, z)+\frac{K(u, z)-K(0, z)}{u}\right) .
$$

We solve this equation using the quadratic method, due to Tutte, as described in [11, p. 515]. Doing so yields an expression for $K(0, z)$ (see [2] for details).

Finally, to find $L(z)$, observe that for any $(D, \alpha) \in \mathcal{L}_{n}$, the path $D$ can be decomposed uniquely as $D=$ $e A_{1} s e A_{2} s \ldots$, where each $A_{j}$ is a Dyck path, and if we let $\alpha^{(j)}$ be its sequence of labels decreased by one, then $\left(A_{j}, \alpha^{(j)}\right)$ is an arbitrary element of $\mathcal{K}$ with $\alpha_{0}^{(j)}=0$. It follows that $L(z)=1 /(1-z K(0, z))$, which gives Eq. (1).

To find the asymptotic behavior of the coefficients, note that the singularity of $L(z)$ nearest to the origin is a branch point at $z=1 / 12$. By [11, Corollary VI.1], its coefficients satisfy Eq. (2).

It is interesting to observe that the generating function in Theorem 4.1 is algebraic, in contrast with the fact that the generating function for 123-avoiding matchings is D-finite but not algebraic [12, 7]. Compare also the growth rate in Eq. (2) with $\left|\mathcal{M}_{n}(123)\right|=C_{n+2} C_{n}-C_{n+1}^{2} \sim \frac{24}{\pi n^{5}} 16^{n}$.

\subsection{2-avoiding partitions}

A refinement of the methods from Section 4.1 can be used to enumerate 312-avoiding partitions, or equivalently, 231-avoiding ones. For any pattern $\tau$, the set of $\tau$-avoiding set partitions can be generated from the set of all $\tau$-avoiding matchings as follows. Given a matching $M$, one can first choose, for each closer immediately followed by an opener, either to merge them into one transitory vertex or to leave them as they are; then one can insert singleton vertices in any position. If we let val $(M)$ denote the number of closers immediately followed by openers in $M$ (we call these valleys of $M$ ), and $A(v, z)=\sum_{n \geq 0} \sum_{M \in \mathcal{M}_{n}(\tau)} u^{\operatorname{val}(M)} z^{n}$ is the generating function for $\tau$-avoiding matchings with respect to the number of valleys, then

$$
\sum_{n \geq 0}\left|\mathcal{P}_{n}(\tau)\right| z^{n}=\frac{1}{1-z} A\left(\frac{1}{z}, \frac{z^{2}}{(1-z)^{2}}\right)
$$

If two patterns satisfy $\sigma \sim \tau$, then $\left|\mathcal{M}_{F}(\sigma)\right|=\left|\mathcal{M}_{F}(\tau)\right|$ for every $F$, and so the above generating function $A(v, z)$ is the same for $\sigma$-avoiding as for $\tau$-avoiding matchings. It follows that $\left|\mathcal{P}_{n}(\sigma)\right|=$ $\left|\mathcal{P}_{n}(\tau)\right|$ for all $n$. In particular, since $312 \sim 231$, we have $\left|\mathcal{P}_{n}(312)\right|=\left|\mathcal{P}_{n}(231)\right|$.

Theorem 4.2 The generating function $B(z)=\sum_{n \geq 0}\left|\mathcal{P}_{n}(312)\right| z^{n}$ for 312-avoiding partitions is a root of the cubic polynomial

$$
\begin{aligned}
(z-1)\left(5 z^{2}-2 z+1\right)^{2} B^{3}+ & \left(-9 z^{5}+54 z^{4}-85 z^{3}+59 z^{2}-14 z+3\right) B^{2} \\
& +\left(-9 z^{4}+60 z^{3}-64 z^{2}+13 z-3\right) B+\left(-9 z^{3}+23 z^{2}-4 z+1\right) .
\end{aligned}
$$

The asymptotic behavior of its coefficients is given by

$$
\left|\mathcal{P}_{n}(312)\right| \sim \delta n^{-5 / 2} \rho^{n}
$$


where

$$
\rho=\frac{3(9+6 \sqrt{3})^{1 / 3}}{2+2(9+6 \sqrt{3})^{1 / 3}-(9+6 \sqrt{3})^{2 / 3}} \approx 6.97685
$$

and $\delta \approx 0.061518$.

Proof sketch: To apply Eq. (3), we need to count 312-avoiding matchings while keeping track of the number of closers immediately followed by an opener. Via the bijection $\Pi \circ \kappa: \mathcal{M}_{n}(312) \rightarrow \mathcal{L}_{n}$, this is equivalent to counting labeled paths in $\mathcal{L}_{n}$ with respect to the number of valleys. Proceeding as in the proof of Theorem 4.1 the generating function $K(u, v, z)$ for paths in $\mathcal{K}$ that refines $K(u, z)$ by marking the number of valleys with the variable $v$ satisfies

$$
K(u, v, z)=1+z(v K(u, v, z)-v+1)\left(2 K(u, v, z)+u K(u, v, z)+\frac{K(u, v, z)-K(0, v, z)}{u}\right) .
$$

Applying the quadratic method, we obtain an expression for $K(0, v, z)$. Then, letting $L(v, z)$ be the generating function for paths in $\mathcal{L}_{n}$ where $v$ marks the number of valleys, we have

$$
L(v, z)=\frac{1 / v}{1-v z K(0, v, z)}-\frac{1}{v}+1 .
$$

Using now Eq. (3) to relate $B(z)$ and $L(v, z)$, it follows that $B(z)$ is a root of the polynomial (4).

To describe the asymptotic growth of its coefficients, we use the method described in [11, Section VII.7.1] to compute the singularities of algebraic functions (see [2] for details).

Again, the generating function in Theorem 4.2 is algebraic, in contrast with the fact that the generating function for 123-avoiding (namely, 3-noncrossing) partitions is D-finite but not algebraic [5]. Compare also Eq. (5) with the growth of the number of 3-noncrossing partitions [5], given by

$$
\left|\mathcal{P}_{n}(123)\right| \sim \frac{3^{9} 5 \sqrt{3}}{2^{5} \pi} \frac{9^{n}}{n^{7}} .
$$

\subsection{An application to 1342-avoiding permutations}

The method involving labeled Dyck paths that we have developed to enumerate 312-avoiding matchings can be used to recover the following generating function due to Bóna [4] for the number of 1342-avoiding permutations (which, by symmetry, equals the number of 3124-avoiding ones).

Theorem $4.3([4])$

$$
\sum_{n \geq 0}\left|\mathcal{S}_{n}(1342)\right| z^{n}=\frac{32 z}{1+20 z-8 z^{2}-(1-8 z)^{3 / 2}} .
$$

Bóna [4] obtained this formula by constructing a bijection between so-called indecomposable 1342avoiding permutations and certain labeled trees, called $\beta(0,1)$-trees. He then used the fact that the generating function for $\beta(0,1)$-trees had already been found by Tutte [20]. Our approach provides a more direct method to enumerate 1342 -avoiding permutations without using $\beta(0,1)$-trees.

Denote by $\mathcal{R}_{n}^{\times}(312)$ the set of placements $(R, F) \in \mathcal{R}_{n}(312)$ with the property that $F$ is the smallest Ferrers board that contains $R$. There is a straightforward bijection $\chi: \mathcal{S}_{n}(3124) \rightarrow \mathcal{R}_{n}^{\times}(312)$ defined 
by $\chi(\pi)=\left(R_{\pi}, F_{\pi}\right)$, where $R_{\pi}$ is the placement consisting of the squares $(i, \pi(i))$ for $1 \leq i \leq n$, and $F_{\pi}$ is the smallest board containing $R_{\pi}$. To enumerate $\mathcal{R}_{n}^{\times}(312)$, we use the fact (proved in [2]) that the image of the map $\Pi: \mathcal{R}_{n}(312) \rightarrow \mathcal{L}_{n}$, when restricted to $\mathcal{R}_{n}^{\times}(312)$, is the set of labeled Dyck paths $(D, \alpha) \in \mathcal{L}_{n}$ such that for every peak $V_{i}$, the labels around it satisfy $\alpha_{i-1}=\alpha_{i}+1=\alpha_{i+1}$. We denote this set by $\mathcal{L}_{n}^{\times}$.

Using the same framework as in the proof of Theorem 4.1 , we can obtain the generating function for these paths. Let $\mathcal{K}_{n}^{\times}$be the set of labeled Dyck paths $(D, \alpha)$ that have the diagonal property, satisfy $\alpha_{2 n}=$ 0 , and such that $\alpha_{i-1}=\alpha_{i}+1=\alpha_{i+1}$ if $V_{i}$ is a peak. Letting $K^{\times}(u, z)=\sum_{n \geq 0} \sum_{(D, \alpha) \in \mathcal{K}_{n}^{\times}} u^{\alpha_{0}} z^{n}$ be the generating function for such paths according to the value of the first label, we obtain

$$
K^{\times}(u, z)=1+z K^{\times}(u, z)\left(K^{\times}(u, z)+(u+1)\left(K^{\times}(u, z)-1\right)+\frac{K^{\times}(u, z)-K^{\times}(0, z)}{u}\right) .
$$

The quadratic method yields a formula for $K^{\times}(0, z)$, from where

$$
\sum_{n \geq 0}\left|\mathcal{S}_{n}(3124)\right| z^{n}=\sum_{n \geq 0}\left|\mathcal{L}_{n}^{\times}\right| z^{n}=\frac{1}{1-z K^{\times}(0, z)}=\frac{32 z}{1+20 z-8 z^{2}-(1-8 z)^{3 / 2}} .
$$

\section{Pairs of patterns}

Tab. 1 summarizes the results from the full paper [2] on matchings and set partitions that avoid a pair of patterns of length 3. The notions of pattern-avoidance and shape-Wilf-equivalence defined in Section 2 have a straightforward generalization to pairs of patterns. We establish that the 15 pairs of patterns in $\mathcal{S}_{3}$ are partitioned into 7 shape-Wilf-equivalence classes. Further, we provide enumeration results for

\begin{tabular}{|c|c|c|c|c|c|c|c|c|}
\hline & Class & Matchings & Set partitions \\
\hline & 132 & 213 & 231 & 312 & 321 & \multirow{2}{*}{ I } & 4 & $2-3 z+z^{2}-z \sqrt{1-6 z+z^{2}}$ \\
\hline 123 & VI & $\mid \mathrm{I}$ & \begin{tabular}{|c|c|}
-51 \\
II
\end{tabular} & \begin{tabular}{|l|l|} 
III \\
\end{tabular} & \begin{tabular}{|l|l|} 
IV \\
\end{tabular} & & $3+\sqrt{1-8 z}$ & $2\left(1-3 z+3 z^{2}\right)$ \\
\hline 132 & & $\mathrm{I}$ & I & $\mathrm{I}$ & VII & II, III & Solutions of a cubic & Solutions of a cubic \\
\hline 213 & & & $\mathrm{I}$ & $\mathrm{I}$ & $\mathrm{V}$ & \multirow{2}{*}{ IV } & $1-5 z+2 z^{2}$ & $1-10 z+32 z^{2}-37 z^{3}+12 z^{4}$ \\
\hline 231 & & & & $\mathrm{I}$ & $\mathrm{I}$ & & $\overline{1-6 z+5 z^{2}}$ & $\overline{(1-z)\left(1-10 z+31 z^{2}-30 z^{3}+z^{4}\right)}$ \\
\hline \multirow[t]{2}{*}{312} & & & & & I & V & Functional equation & Unknown \\
\hline & & & & & & VI, VII & Unknown & Unknown \\
\hline
\end{tabular}
matchings and set partitions avoiding a pair of patterns in many cases.

Tab. 1: The 7 shape-Wilf-equivalence classes of pairs of patterns, and a summary of our enumeration results.

\section{References}

[1] J. Backelin, J. West and G. Xin, Wilf-equivalence for singleton classes, Adv. in Appl. Math. 38 (2007), $133-148$. 
[2] J. Bloom and S. Elizalde, Pattern avoidance in matchings and partitions, preprint, arXiv: 1211.3442 .

[3] J. Bloom and D. Saracino, A simple bijection between 231-avoiding and 312-avoiding placements, $J$. Combin. Math. Combin. Comput., to appear.

[4] M. Bóna, Exact enumeration of 1342-avoiding permutations: a close link with labeled trees and planar maps, J. Combin. Theory Ser. A 80 (1997), 257-272.

[5] M. Bousquet-Mélou and G. Xin, On partitions avoiding 3-crossings, Sém. Lothar. Combin. 54 (2005/07), Art. B54e, 21 pp.

[6] S. Burrill, S. Elizalde, M. Mishna and L. Yen, A generating tree approach to $k$-nonnesting partitions and permutations, preprint, arXiv:1108.5615.

[7] W. Chen, E. Deng, R. Du, R. Stanley and C. Yan, Crossings and nestings of matchings and partitions, Trans. Amer. Math. Soc. 359 (2007), 1555-1575.

[8] A. Claesson, V. Jelínek and E. Steingrímsson, Upper bounds for the Stanley-Wilf limit of 1324 and other layered patterns, J. Combin. Theory Ser. A 119 (2012), 1680-1691.

[9] A. de Mier, $k$-noncrossing and $k$-nonnesting graphs and fillings of Ferrers diagrams, Combinatorica 27 (2007), 699-720.

[10] S. Elizalde and I. Pak, Bijections for refined restricted permutations, J. Combin. Theory Ser. A 105 (2004), 207-219.

[11] P. Flajolet and R. Sedgewick, Analytic Combinatorics, Cambridge University Press, Cambridge, 2009.

[12] D. Gouyou-Beauchamps, Standard Young tableaux of height 4 and 5, European J. Combin. 10 (1989), 69-82.

[13] V. Jelínek, Dyck paths and pattern-avoiding matchings, European J. Combin. 28 (2007), 202-213.

[14] M. Klazar, On abab-free and abba-free set partitions, European J. Combin. 17 (1996) 53-68.

[15] C. Krattenthaler, Permutations with restricted patterns and Dyck paths, Adv. in Appl. Math. 27 (2001), 510-530.

[16] C. Krattenthaler, Growth diagrams, and increasing and decreasing chains in fillings of Ferrers shapes, Adv. in Appl. Math. 37 (2006), 404-431.

[17] B. E. Sagan, Pattern avoidance in set partitions, Ars Combin. 94 (2010), 79-96.

[18] Z. Stankova, Shape-Wilf-Ordering on Permutations of Length 3, Elec. J. Combin. 14 (2007), \#R56.

[19] Z. Stankova and J. West, A new class of Wilf-equivalent permutations, J. Alg. Combin. 15 (2002), 271-290.

[20] W. T. Tutte, A census of planar maps, Canad. J. Math. 15 (1963), 249-271. 